\title{
MODELING ETHICS: \\ REFLECTIONS AT THE INTERSECTION OF ETHICS AND SIMULATION
}

\author{
Wesley J. Wildman \\ Boston University and the Center for Mind and Culture \\ 566 Commonwealth Avenue, Suite M-2 \\ Boston, MA 02215, USA \\ wwildman@bu.edu
}

\begin{abstract}
The rise of computer simulation provokes a diverse range of ethical questions, which computational modelers are striving to navigate. Computer simulation can also be turned to the analysis of ethical themes such as the origins and spread of social norms and the processes of scientific research and technological development; these lines of inquiry have the potential to change the way the philosophical field of ethics conceives itself. In this two-way traffic between simulation and ethics, two imperatives arise: the ethical obligation to apply computer simulations to urgent social problems and the obligation for philosophers to be prepared to offer ethical analyses of every new application of computer simulation to human affairs. This paper discusses these four lines of interaction, argues that close connections between simulation and ethics will only become more important in the future as the power and influence of computational simulation increases, and advances several practical suggestions.
\end{abstract}

Keywords: simulation, social simulation, human simulation, ethics, philosophy

\section{INTRODUCTION}

Every complex technological domain generates debates over ethics. With enough time, a parallel expertlevel, systematic discussion of ethics within the domain of technological activity arises. This has happened, for example, in medicine, biotechnology, business, and ecology, where the ethics conversations are advanced and well organized. In newer technological domains such as artificial intelligence, the ethics debates are intense but only now becoming somewhat systematic (see Himma \& Tavani 2008). In modeling $\&$ simulation $(M \& S)$, the ethics enterprise is even newer and the key debates themselves are still emerging.

Of all these fields, M\&S is almost unique in its ability to turn the tables on ethics and make the practice of ethics and human morality itself an object of study, even as ethical analysis of M\&S activities remains a high priority. This essay focuses on this fascinating two-way interaction between M\&S and ethics, including the imperatives that arise on both sides in the wake of this interaction (Figure 1).

For the purposes of this essay, morality refers to appraisals of human behavior generally while ethics refers to intellectual reflection on specific domains of professional activity or on morality taken as a whole. Thus, we might make a moral appraisal of someone's behavior as laid out for us in a criminal court case, whereas we would make an ethical appraisal of the justice system itself. Again, we may or may not monitor our moral responses to subject matters we work on when constructing computational simulations but hopefully we are permanently mindful of our ethical obligations as M\&S professionals. 


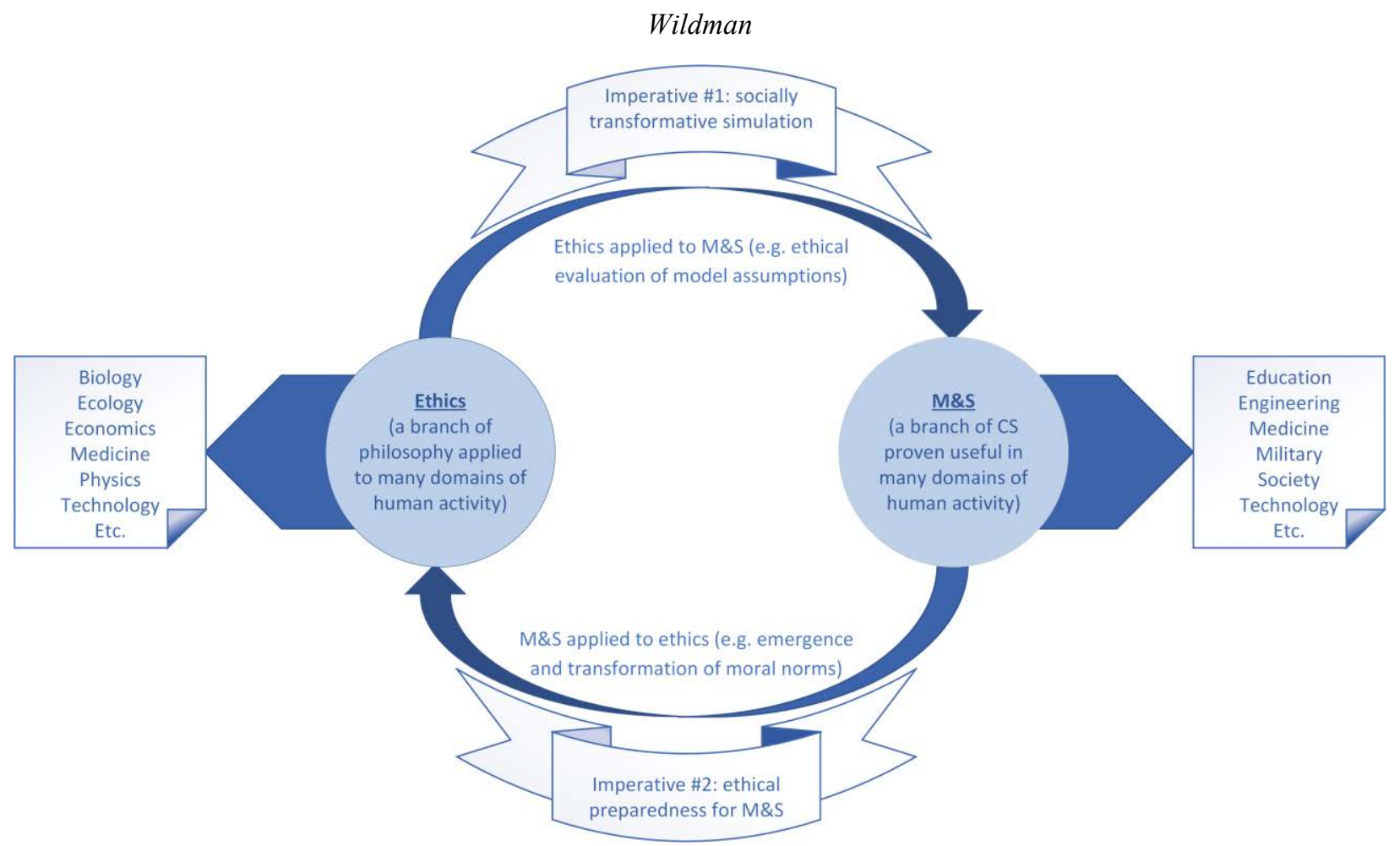

Figure 1: Structure of the paper tracks salient relationships between Ethics and M\&S: the top arrow corresponds to sections $2 \& 4$ while the bottom arrow corresponds to sections $3 \& 5$.

\section{ETHICS OF SIMULATION}

M\&S activities are expanding rapidly in every aspect of human life, from physics to biology to engineering, and from sociology to business to policy. As a consequence, the M\&S culture tends to be exploratory and fast-changing, and the ethical controversies are still forming. Nevertheless, certain key ethical challenges and postures have been identified both at the level of professional identity and at the level of content.

At the level of a professional identity, the Society for Modeling \& Simulation International has produced the "Simulationist Code of Ethics" (The Society for Modeling \& Simulation International 2018, reproduced in Appendix A; also see Oren 2002 and the discussion in Duran 2018, ch. 7). This document is general in application and broad in scope, dealing with professional obligations in five categories: (1) personal development and profession, (2) professional competence, (3) trustworthiness, (4) property rights and due credit, and (5) compliance with the code, which lays out self-reflexive ethical norms for how to regard and use the code itself. It is crafted with a certain degree of flexibility so that it can remain relevant even as simulation technologies and professional activities change over time.

At the level of specific domains of ethical concern, several have come to the fore. First, safety concerns are critical because lives are often at stake. Will a simulated bridge that withstands a simulated category-5 hurricane actually survive when the design takes real-world form? Privacy issues are also important, as they are in most information-rich technology domains. In the non-simulation modeling realm, should web-based advertising engines be allowed to predict my interests based on a machine-learning model informed by my browser search history (which is currently routine and rarely a matter for user choice)? And in the simulation realm, should we model an artificial society populated with AI agents using data on specific real-world individuals (which our group did in Shults et al. 2018)? There are more recondite ethical puzzles concerning our moral obligations to simulated AIs, now and in the future, and a host of similar questions. At least as pressing as safety, privacy, and AI concerns are the social implications of the accelerating 
employment of M\&S in social policy and planning (see Desai 2012, Gilbert et al. 2018). That's where I focus attention here as this issue has far-reaching implications for all future human civilizations.

Simulation results are assumption-relative, everywhere and always. If assumptions are inappropriate, results will be worthless - garbage in, garbage out. Even when assumptions are appropriate, however, great caution is required when interpreting results from a computational simulation. The small corner of M\&S that deals with social policy - so-called policy simulation, a branch of social simulation - is particularly instructive in this regard.

A computational simulation of a policy intervention furnishes a basis for evaluating the policy of interest by comparing the social system before and after the imagined policy is introduced. We build a model without the envisaged policy in place and validate it against known data to gain confidence in its accuracy. Then we can reasonably hope that the same model, minimally modified to incorporate the new policy, will tell us something accurate about what would happen if we were to implement the policy in the real world.

This is an obvious procedure, akin to using simulation to evaluate a bridge design or a distribution network under stress. But there's a critical difference: policy simulation involves modeling complex adaptive social systems, which are difficult to predict in real life because they are vulnerable to perturbation from individual actors with great charisma or creativity, by chance events, or by unforeseen technologies. For instance, global economic systems were completely transformed by the introduction of the computer, with a cascade of downstream policy-related complications. These complications include the child trafficking epidemic, facilitated by social media, which is a large (USD\$32 billion) and apparently rapidly growing segment of organized crime (United Nations Office on Drugs and Crime 2012). Nobody was anticipating the child trafficking disaster when they were formulating policies about internet usage a few decades ago. And now that we have identified massively increased trafficking as a side effect, attempts to change policy to curb the problem are met with stout resistance not only from corrupt government officials but also from freespeech advocates who don't want anything to curtail the free exchange of ideas in cyberspace.

The Simulationist Code of Ethics prudently urges simulationists to "Provide full disclosure of system design assumptions and known limitations and problems to authorized parties," to "Be explicit and unequivocal about the conditions of applicability of specific models and associated simulation results," and to "Caution against acceptance of modeling and simulation results when there is insufficient evidence of thorough validation and verification." That arguably covers ethical problems associated with policy simulation in a general way suited to a professional code of ethics and open to a wide variety of applications of M\&S. In practice, however, social simulations sometimes suggest policy applications that almost everyone would resist, so additional specificity might be useful.

For example, our simulation of conditions for longevity of violent extremist groups yields a criterion for targeted assassination of group leaders by those opposing the extremists' goals (Wildman \& Sosis 2011). And our simulation of mutually escalating religious violence suggests that one way to reduce social tension is partial genocide or forced migration of a minority population to decrease frequency of contact (Shults et al. 2019). Both simulations were developed for social-science investigation, not policy applications, but the policy implications are difficult to miss. Moreover, these policy implications are not ridiculous fantasies, since all of them have actually been implemented in the recent past, even in so-called developed nations.

What happens when a verified and validated simulation with policy implications of this kind finds its way into the hands of people looking for a convenient rationalization of their social agenda? Worse, in an era when people learn to trust policy simulations, what happens when a poorly verified and validated simulation, or one that is deliberately rigged, is cynically deployed to support an ideological posture? In engineering simulations, the bridge falls and the distribution network fails, which is bad enough. But in policy applications, the scale of the ensuing moral disaster could be truly vast.

Part of the solution here is to hold people - politicians and policy professionals - responsible for social policies, regardless of the technical aids employed to analyze policy alternatives (e.g. policy simulations, 
spreadsheets, demographic projections, etc.). Correspondingly, simulation professionals must regard the simulations they build as ways to generate insight capable of guiding policy professionals rather than as a substitute for careful policy analysis. Simulation professionals should teach policy professionals to regard simulations as insight-generators that should only ever function in an advisory capacity alongside all of the more familiar considerations that go into the creation of sound policy.

In previous work, I have helped lay out a systematic schema for analyzing the ethics of simulation (Shults \& Wildman 2019). This is a way of thinking ethically about simulation activities, embracing (1) philosophical metaethics to generate clear ethical concepts and analytical frameworks, (2) scientific metaethics to link ethical reasoning with accurate empirical information about human behavior, and (3) practical metaethics to deploy the first two principles in such a way as to attack the perennial problems of ethical confusion and evasion of responsibility. This is a robust framework for ethical thinking but, like the simulationist's professional code of ethics, it is also very general. When applied to policy simulation, for example, the third point needs to be spelled out in more specific terms: human beings are always accountable for social policies and simulations should be regarded strictly as insight-generating adjuncts to policy reasoning. Sadly, this specific application of practical metaethics is likely to be neglected or ignored repeatedly in the future. One way of remembering it, and the warning it conveys, would to build it into the simulationist's professional code of ethics.

\section{SIMULATION OF ETHICS}

With that sobering specter of simulation-abetted policy disasters in mind, perhaps we should ask what we are doing when we do ethics. The history of our species makes it clear that we are not very good at ethical reasoning, we use it to rationalize our moral instincts regardless of which way they point, and our moral compass seems to spin more freely than our cosmologized stories about the origins of morality suggest that it should. There's the mystery of how moral norms are generated and propagated through a human culture, seemingly becoming invisible rules that we internalize so completely we do not even notice them in operation - perfect for the untraceable exercise of social control. And then there's the puzzle of moral enforcement, including the way we rely on invisible monitoring beings (angels, demons, jinns, gods, ghosts, ancestors) to handle moral evaluation of people's internal mental states and outward behavior that we ourselves may not observe, all associated with stories about supernaturally delivered moral punishments and rewards. Here again we have a process to which our evolved minds apparently dispose us, one that supports social control.

Scientific study of human morality is in a period of rapid transformation, on several fronts simultaneously. Here's a short list of discoveries from a variety of disciplinary perspectives.

- Social psychology applied to moral intuitions and behavior is revolutionizing philosophical and practical ethics with its evolutionary framing of the phenomena just described backed by ingenious experiments showing how various elements of moral behavior actually operate, at least to the extent that this can be determined in lab settings, and how moral reasoning relates to political ideology (e.g. Haidt 2001, Haidt 2007, Graham \& Haidt 2009).

- Social theory has unmasked the dynamics of the social construction of reality by which distinctive elements of a culture can be externalized and rendered as objective norms for that culture's inhabitants, after which those norms are internalized through learning and training, much as a native language is internalized for most young humans (e.g. Berger \& Luckmann 1967). This field has also investigated the key roles religion plays in the process of socially constructing social worlds (e.g. Berger 1967).

- Cultural anthropology and social history have uncovered the moral worlds of hundreds of human cultures, past and present. This prodigious work manifests the cultural diversity of human morality (and the less adaptable species-wide moralities of other primates) while also identifying patterns of convergence in moral norms apparently rooted in the dynamics of evolved moral minds solving 
problems that recur across cultures, from sex management and child-rearing to reinforcement of group loyalty and fairness of economic exchange.

- Twin studies have shown that some aspects of morality are heritable to a fairly strong degree, such as authoritarianism (e.g. Bouchard et al. 1999). Personality psychology has uncovered correlates between personality and moral preferences that begin to explain why human beings have different moral responses to the same circumstances (e.g. Mudrack 2006). Developmental psychology has identified several distinct phases in the development of human moral capacities that can help us understand age-appropriate forms of moral training (e.g. Piaget 1932, Kohlberg 1958, 1984). Psychology of religion has started to uncover the way prized moral virtues such as humility and gratitude are cultivated in life-long processes of group-supported ventures of individual development (e.g. Schnitker \& Emmons 2017, Paine \& Sandage 2015).

A robust understanding of human morality requires synthesizing all these powerful disciplinary insights into a unified theoretical edifice that can be tested as a whole, rather than piece-wise, fragment by fragment, discipline by discipline. Environmental cueing, social context, and interaction effects almost certainly matter in human morality so treating all elements together is critical for overcoming the problems of ecological validity that plague lab-based experiments and make experimental replication such a difficult challenge (Open Science Network 2018).

This is where M\&S shines as a method. M\&S enables us to implement syntheses of competing or complementary part-theories as causal architectures for a virtual complex system that we can use to model a real-world complex system. For this reason, $M \& S$ has enormous potential as an adjunct to theory construction and experiment, helping us to discover how the varied disciplinary insights fit together into a larger system with looping interactions that reinforce and dampen system dynamics. This is why M\&S has been called the third pillar of science, alongside theory and experiment (discussed in Tolk 2015), as well as the foundation for generative social science (Epstein \& Axtel 1996).

The study of human morality is ripe for the application of M\&S methods. This potential has been noticed by a number of researchers and the computational simulation of human and primate morality. Of course, simulation has been used for ethical training in health care and a variety of other fields. Here, though, I'm thinking of simulation research into how human morality works rather than merely using simulation to inculcate professional ethical behavior. This type of research has yielded valuable results already in domains as diverse as criminology (e.g. Birks et al. 2012), norm-generation (e.g. Conte et al. 2014, Muldoon et al. 2014), policy norms (e.g. Dignum 2010), marketing behavior (e.g. Hill \& Watkins 2007), social conflict (e.g. Lemos 2017), primate friendship and post-conflict reconciliation (e.g. Puga-Gonzalez \& Sueur 2017, Puga-Gonzalez et al. 2014), social segregation (e.g. Schelling 1971), and terror management (e.g. Shults et al. 2017).

What's missing is a more comprehensive approach that integrates everything we have learned about human morality from individual disciplines (sketched above) within an artificial society constituted by AI agents with minds sufficiently complex that the dynamics of existing scientific insights into human morality can be fairly represented. That is one of the most important challenges facing the simulation of ethics and one with almost unlimited promise for understanding how morality works in the human and other primate species.

\section{IMPERATIVE \#1: SOCIALLY TRANSFORMATIVE SIMULATION}

Nowhere in the Society for Modeling \& Simulation International's Simulationist Code of Ethics does it say anything like "simulators should strive to improve society." This is understandable: a professional code of ethics focuses on professional behaviors rather than high-level goals and motivations. Nevertheless, I see the absence of this statement or something akin to it as a problem, or at the very least a missed opportunity. 
Computer engineers and simulationists sometimes understand themselves merely as tool wielders hired out to clients to achieve client goals. But this self-understanding threatens to replicate within computer engineering the severe ethical problems that arose within physics and biology during the twentieth century, especially in relation to weapons of mass destruction and environmental catastrophes. Some scientists declared that technological application of their research is a political and social issue that lies beyond the scope of scientific responsibility. To this self-exculpatory move, a group of scientists from the Massachusetts Institute of Technology objected through a now-famous 1968 statement (Union of Concerned Scientists 1968). This statement appeared in the wake of what these MIT scientists felt was abuse of military technology in the Vietnam War, unquestionable ecological disasters such as the Cuyahoga River catching fire in Cleveland, and of course the military use of nuclear weapons in Japan near the end of World War II. Their rousing statement became the founding document of the Union of Concerned Scientists when it was created in 1969, "calling for scientific research to be directed away from military technologies and toward solving pressing environmental and social problems" (Union of Concerned Scientists 2018).

Our ever-increasing reliance on computational simulation technologies to guide human affairs places computer engineers on a collision course with widely prized social values from alleviating needless suffering to efficient use of public resources when implementing social policy. The pitfalls associated with reliance on policy simulation described in the second section are lying in wait for us but we don't need to fall into those traps. We've seen what can happen in other fields already. We need to get out ahead of these problems by doing within M\&S what the Union of Concerned Scientists already did half a century ago within science generally: undertake to focus $M \& S$ on the betterment of society.

Socially transformative simulation is a meaningful professional goal but realizing it critically depends on widening the ethics conversation within the M\&S community so that the root disagreement can be surfaced clearly and discussed. That root disagreement is the same as it has always been: it is the self-exculpatory understanding of simulationists as mere tool wielders on behalf of clients, like carpet cleaners and plumbers, versus the responsibility-taking understanding of simulationists as powerful participants in forging future societies, like politicians and policy planners. Surface the disagreement, debate it publicly, and we have a chance to avoid repeating infamous mistakes of the past. This begins with the training of simulationists, which is still largely an ethics-free zone.

This is the first of two moral imperatives - computational simulation should be dedicated to pro-social ends. As stated, it is not easy to interpret, obviously; for instance, there are always people who think society is improved when a privileged minority sequesters resources and doles them out to the less powerful as the privileged see fit. Without oversimplifying the hermeneutical and implementation challenges, I believe it is critical for the field of M\&S to take a stand on this, just as the Union of Concerned Scientists has already done.

\section{IMPERATIVE \#2: ETHICAL PREPAREDNESS FOR SIMULATION}

The second moral imperative I think we need to discuss bears on the professional obligations of ethicists in light of the rapid development and diversification of simulation activities - ethicists need to think ahead and be ready for every new simulation technology and the associated practical effects. Can ethicists rely on simulationists to adopt the first moral imperative? It is likely that they can trust some but not others, because the underlying dispute about scope of responsibility won't go away - it hasn't gone away in physics or biology, and there's no reason to think it will just vanish in computational engineering. Thus, ethicists need to be on guard, setting forth ethical analyses of simulation activities, sponsoring ethics discussions within the $\mathrm{M} \& \mathrm{~S}$ community, encouraging the adoption of pro-social simulation principles, and publicly resisting applications of simulation technologies that fail to embody those principles.

This is a lot to ask of professional ethicists because few of them know much at all about computational M\&S. It follows that the professional field of philosophical ethics itself needs to organize around new 
information technologies such as M\&S just as it has around weapons of mass destruction, biotechnologies, business ethics, medical ethics, and other domains calling for ethical analysis and oversight. One way to encourage this to happen is to wait until a series of simulation-abetted social policy disasters occurs so that ethicists are motivated to spring into action. A better way is to anticipate these problems and start the conversation between ethicists and computer engineers right now, striving to create a culture of ethical leadership that affords some measure of protection against the disasters we can so easily imagine.

Professional ethicists also need to learn about information technologies such as M\&S, just as medical ethicists learn medicine and business ethicists learn business. Joint degrees or philosophy degrees majoring in both ethics and computer science would be one place to begin. Another starting point is to have computer engineers invite ethicists into their domain to educate them and to benefit from their professional experience. That, of course, is already happening with this essay, written by a philosopher in a computerengineering context that facilitates mutual exchange.

\section{CONCLUSION}

The implications of these two imperatives need to be spelled out and that will take some time. Here are two practical suggestions concerning what taking both seriously could mean for the intersection of ethics and simulation.

First, the simulation profession's codes of ethics might emphasize that responsible simulationists should seek to partner with ethicists to monitor and advise on simulation activities, much as universities require researchers studying human beings to partner with Institutional Review Boards for ethical oversight aimed at protecting human subjects. Just as human-subjects researchers need to take trainings in the history and practice of protecting human subjects, so simulation researchers might be required to take trainings in the negative social effects of high technology including computational $M \& S$ technologies.

Second, the simulation profession's codes of ethics might follow the lead of the Union of Concerned Scientists and explicitly incorporate a statement that the goals and motivations of responsible simulationists should be to improve society and protect vulnerable people. This could be reinforced by publicizing case studies that inspire simulationists to apply their efforts to pro-social goals. That is what the 2018 Winter Simulation Conference was all about - simulation for a noble cause - and we all need a lot more of the same.

\section{ACKNOWLEDGMENTS}

This paper was written while the author was funded on a subcontract from the Modeling Religion in Norway (MODRN) project, which was supported by a grant from The Research Council of Norway (\# 250449). I am grateful to F. LeRon Shults and Patrick Grim for discussions of this paper.

\section{A APPENDIX: "SIMULATIONIST CODE OF ETHICS" FROM THE SOCIETY FOR MODELING \& SIMULATION INTERNATIONAL}

The Society for Modeling \& Simulation International: Simulationist Code of Ethics

\section{Preamble}

Simulationists are professionals involved in one or more of the following areas:

- Modeling and simulation activities.

- Providing modeling and simulation products.

- Providing modeling and simulation services. 


\section{Personal Development and Profession}

As a simulationist I will:

1.1 Acquire and maintain professional competence and attitude.

1.2 Treat fairly employees, clients, users, colleagues, and employers.

1.3 Encourage and support new entrants to the profession.

1.4 Support fellow practitioners and members of other professions who are engaged in modeling and simulation.

1.5 Assist colleagues to achieve reliable results.

1.6 Promote the reliable and credible use of modeling and simulation.

1.7 Promote the modeling and simulation profession; e.g., advance public knowledge and appreciation of modeling and simulation and clarify and counter false or misleading statements.

\section{Professional Competence}

As a simulationist I will:

2.1 Assure product and/or service quality by the use of proper methodologies and technologies.

2.2 Seek, utilize, and provide critical professional review.

2.3 Recommend and stipulate proper and achievable goals for any project.

2.4 Document simulation studies and/or systems comprehensibly and accurately to authorized parties.

2.5 Provide full disclosure of system design assumptions and known limitations and problems to authorized parties.

2.6 Be explicit and unequivocal about the conditions of applicability of specific models and associated simulation results.

2.7 Caution against acceptance of modeling and simulation results when there is insufficient evidence of thorough validation and verification.

2.8 Assure thorough and unbiased interpretations and evaluations of the results of modeling and simulation studies.

\section{Trustworthiness}

As a simulationist I will:

3.1 Be honest about any circumstances that might lead to conflict of interest.

3.2 Honor contracts, agreements, and assigned responsibilities and accountabilities.

3.3 Help develop an organizational environment that is supportive of ethical behavior.

3.4 Support studies which will not harm humans (current and future generations) as well as environment.

\section{Property Rights and Due Credit}

As a simulationist I will:

4.1 Give full acknowledgement to the contributions of others.

4.2 Give proper credit for intellectual property.

4.3 Honor property rights including copyrights and patents.

4.4 Honor privacy rights of individuals and organizations as well as confidentiality of the relevant data and knowledge.

\section{Compliance with the Code}

As a simulationist I will:

5.1 Adhere to this code and encourage other simulationists to adhere to it.

5.2 Treat violations of this code as inconsistent with being a simulationist. 
5.3 Seek advice from professional colleagues when faced with an ethical dilemma in modeling and simulation activities.

5.4 Advise any professional society which supports this code of desirable updates.

\section{REFERENCES}

Berger, P. L., and T. Luckmann. 1967. The Social Construction of Reality: A Treatise in the Sociology of Knowledge. Garden City, N.Y: Doubleday.

Berger, P. 1967. The Sacred Canopy: Elements of a Sociological Theory of Religion. Garden City, NY: Doubleday.

Birks, D., M. Townsley, and A. Stewart. 2012. "Generative Explanations of Crime: Using Simulation to Test Criminological Theory." Criminology 50 (1): 221-254.

Bouchard, T., M. McGue, D. Lykken, and A. Tellegen. 1999. "Intrinsic and Extrinsic Religiousness: Genetic and Environmental Influences and Personality Correlates." Twin Research, 2(2), 88-98.

Conte, R., G. Andrighetto, and M. Campennì. 2014. Minding Norms: Mechanisms and Dynamics of Social Order in Agent Societies. Ocford, UK: Oxford University Press.

Desai, A. 2012. Simulation for Policy Inquiry. New York: Springer Science

Dignum, V., F. Dignum, S. A. Osinga, and G. J. Hofstede. 2010. "Normative, Cultural and Cognitive Aspects of Modelling Policies.” In Proceedings - Winter Simulation Conference, 720-732.

Duren, J. M. 2018. Computer Simulations in Science and Engineering: Concepts-Practices - Perspectives. The Frontiers Collection. New York: Springer.

Epstein, J. M. and Axtell, R. 1996. Growing Artificial Societies: Social Science from the Bottom Up. Cambridge, MA: The MIT Press.

Gilbert, N., P. Ahrweiler, P. Barbrook-Johnson, K. P. Narasimhan, and H. Wilkinson. 2018. "Computational Modelling of Public Policy." Journal of Artificial Societies and Social Simulation 21(1), 1-19.

Graham, J., J. Haidt, and B. Nosek. 2009. "Liberals and Conservatives Use Different Sets of Moral Foundations." Journal of Personality and Social Psychology 96, 1029-1046.

Haidt, J. 2001. "The Emotional Dog and Its Rational Tail: A Social Intuitionist Approach to Moral Judgment." Psychological Review 108, 814-834

Haidt, J. 2007. "The New Synthesis in Moral Psychology” Science 316, 998-1002.

Hill, R. P. and A. Watkins. 2007. "A Simulation of Moral Behavior within Marketing Exchange Relationships." Journal of the Academy of Marketing Science 35(3), 417-29.

Himma, K. E. and H. T. Tavani (eds.). 2008. The Handbook of Information and Computer Ethics. Hoboken, NJ: John Wiley \& Sons.

Kohlberg, L. 1958. "The Development of Modes of Thinking and Choices in Years 10 to 16." Ph.D. Dissertation, University of Chicago.

Kohlberg, L. 1984. "The Psychology of Moral Development: The Nature and Validity of Moral Stages (Essays on Moral Development, Volume 2). New York: Harper \& Row.

Lemos, C. M. 2017. Agent-Based Modeling of Social Conflict: From Mechanisms to Complex Behavior. New York: Springer.

Muldoon, R., C. Lisciandra, C. Bicchieri, S. Hartmann, J. Sprenger. 2014. "On the Emergence of Descriptive Norms." Politics, Philosophy \& Economics 13(1), 3-22.

Mudrack, P. E. 2006. "Moral Reasoning and Personality Traits.” Psychological Reports 98(3), 689-698.

Open Science Network. 2018. https://www.osn.global/. Accessed November 24, 2018. 
Oren, T. I., M. S. Elzas, I. Smit, and L. G. Birta. 2002. "Code of Professional Ethics for Simulationists.” In Summer Computer Simulation Conference, 434-435. Society for Computer Simulation International.

Paine, D. and S. J. Sandage. 2015. "Humility as a Psychotherapeutic Virtue: Spiritual, Philosophical, and Psychological Foundations." Journal of Spirituality in Mental Health 17(1), 3-25.

Piaget, J. 1932. The Moral Judgment of the Child. London, UK: Kegan, Paul, Trench, Trubner \& Co.

Puga-Gonzalez, I. and C. Sueur. 2017. "Friendships and Social Networks in an Individual-Based Model of Primate Social Behaviour." Journal of Artificial Societies and Social Simulation 20(3).

Puga-Gonzalez, I., M. Butovskaya, B. Thierry, C. K. Hemelrijk, and S. C. Pratt. 2014. "Empathy versus Parsimony in Understanding Post-Conflict Affiliation in Monkeys: Model and Empirical Data." PLoS ONE 9(3).

Schelling, T. C. 1971. "Dynamic Models of Segregation.” Journal of Mathematical Sociology 1(2), 143186.

Schnitker, A. A. and R. A. Emmons. 2017. "The Psychology of Virtue: Integrating Positive Psychology and the Psychology of Religion." Psychology of Religion and Spirituality 9(3), 239-241.

Shults, F. L, J. E. Lane, S. Y. Diallo, C. J. Lynch, W. J. Wildman, and R. Gore. 2017. "Modeling Terror Management Theory: Computer Simulations of the Impact of Mortality Salience on Religiosity." Religion, Brain \& Behavior 7(3), 1-24.

Shults, F. L., R. Gore, C. Lemos, and W. J. Wildman. 2018. "Why do the Godless Prosper? Modeling the Cognitive and Coalitional Mechanisms that Promote Atheism." Psychology of Religion and Spirituality $10(3), 218-228$.

Shults, F. L., R. Gore, W. J. Wildman, C. J. Lynch, J. E. Lane, and M. D. Toft. 2019. “A Generative Model of the Mutual Escalation of Anxiety Between Religious Groups." Journal of Artificial Societies and Social Simulation 22(1).

Shults, F. L. and W. J. Wildman. 2019. "Ethics, Computer Simulation, and the Future of Humanity." In Human Simulation, edited by S. Diallo, W. Wildman, F. L. Shults, and A. Tolk. Berlin: Springer.

The Society for Modeling \& Simulation International. 2018. "Simulationist Code of Ethics." http://scs.org/ethics/. Accessed November 24, 2018.

Tolk, A. 2015. "Learning Something Right from Models That Are Wrong: Epistemology of Simulation." In Concepts and Methodologies for Modeling and Simulation, edited by L. Yilmaz, 87-106. New York: Springer.

Union of Concerned Scientists. 1968. "Founding Document: 1968 MIT Faculty Statement." https://www.ucsusa.org/about/founding-document-1968.html. Accessed November 24, 2018.

Union of Concerned Scientists. 2018. "Our History and Accomplishments." https://www.ucsusa.org/about/history-of-accomplishments.html. Accessed November 24, 2018.

United Nations Office on Drugs and Crime. 2012. "Human Trafficking: Organized Crime and the Multibillion Dollar Sale of People." http://www.unodc.org/unodc/en/frontpage/2012/July/humantrafficking_-organized-crime-and-the-multibillion-dollar-sale-of-people.html. Accessed November 24, 2018.

Wildman, W. J., R. Sosis. 2011. "Stability of Groups with Costly Beliefs and Practices." Journal of Artificial Societies and Social Simulation 14(3).

\section{AUTHOR BIOGRAPHY}

Wesley J. Wildman, Ph.D. is Professor of Philosophy, Theology, and Ethics at Boston University and Executive Director of the Center for Mind and Culture in Boston, MA. A philosopher of religion and ethicist specializing in the study of worldviews and related behavior, his research interests include scientific, 
philosophical, and ethical analysis of the human condition, including emerging technologies. For more information, see www.WesleyWildman.com. His email address iswwildman@bu.edu. 